\title{
Definitive chemoradiotherapy has comparable survival outcomes to esophagectomy in patients with clinical T1NOM0 esophageal squamous cell carcinoma: real world data
}

\section{Kentaro Sawada}

Kushiro Rosai Hospital: Kushiro Rosai Byoin https://orcid.org/0000-0001-7952-2765

Hiroki Yukami

National Cancer Center-Hospital East: Kokuritsu Gan Center Higashi Byoin

Saori Mishima

National Cancer Center-Hospital East: Kokuritsu Gan Center Higashi Byoin

Hisashi Fujiwara

National Cancer Center-Hospital East: Kokuritsu Gan Center Higashi Byoin

Tomohiro Kadota

National Cancer Center-Hospital East: Kokuritsu Gan Center Higashi Byoin

Keiichiro Nakajo

National Cancer Center-Hospital East: Kokuritsu Gan Center Higashi Byoin

Yusuke Yoda

National Cancer Center-Hospital East: Kokuritsu Gan Center Higashi Byoin

Masaki Nakamura

National Cancer Center-Hospital East: Kokuritsu Gan Center Higashi Byoin

Hidehiro Hojo

National Cancer Center-Hospital East: Kokuritsu Gan Center Higashi Byoin

Tomonori Yano

National Cancer Center-Hospital East: Kokuritsu Gan Center Higashi Byoin

Takeo Fujita

National Cancer Center-Hospital East: Kokuritsu Gan Center Higashi Byoin

Takashi Kojima

National Cancer Center-Hospital East: Kokuritsu Gan Center Higashi Byoin

Daisuke Kotani ( $\nabla$ dkotani@east.ncc.go.jp )

Department of Gastroenterology and Gastrointestinal Oncology https://orcid.org/0000-0002-4196$555 \mathrm{X}$ 
Keywords: Esophageal Neoplasms, treatment, chemoradiotherapy, esophagectomy, aged

Posted Date: August 30th, 2021

DOI: https://doi.org/10.21203/rs.3.rs-854898/v1

License: (c) (1) This work is licensed under a Creative Commons Attribution 4.0 International License. Read Full License 


\section{Abstract \\ Background}

Recently, the JCOG0502 has shown a comparable efficacy of chemoradiotherapy and esophagectomy in patients with clinical T1NOM0 esophageal squamous cell carcinoma. However, few studies have compared the clinical outcomes of these treatments in esophageal squamous cell carcinoma patients (including elderly patients) based on real-world data.

\section{Methods}

This retrospective study determined real-world outcomes in patients who underwent chemoradiotherapy or esophagectomy, including those with clinical T1NOMO esophageal squamous cell carcinoma, between 2009 and 2017 at the National Cancer Center Hospital East.

\section{Results}

Among a total of 156 patients, 120 and 36 patients underwent esophagectomy and chemoradiotherapy, respectively; 138, 12 and 6 patients had Eastern Cooperative Oncology Group performance status 0, 1, and 2, respectively; and 33 and 123 patients had clinical tumor depth MM-SM1 and SM2-SM3, respectively. In a median follow-up of 72 months, 5-year progression-free survival and overall survival were respectively $77.0 \%$ and $81.5 \%$ in the esophagectomy group and $74.4 \%$ and $82.6 \%$ in the chemoradiotherapy group $(P=0.48$ and,$P=0.89)$. Moreover, no treatment-related death was detected in both groups. In elderly patients (75 years or older), 5-year progression-free survival and overall survival were not significantly different between esophagectomy and chemoradiotherapy groups (5-year progression-free survival: $72.3 \%$ vs. $81.8 \%, P=0.38 ; 5$-year overall survival: $76.9 \%$ vs. $81.8 \%, P=0.59$ ).

\section{Conclusions}

This real-world study confirms the results of a previous clinical trial, and the present findings support chemoradiotherapy as one of the standard treatment options in patients of all ages with clinical T1NOMO esophageal squamous cell carcinoma.

\section{Introduction}

Esophageal cancer is the eighth most common cancer in the world, leading to about a half million of deaths each year [1]. Western populations have a high prevalence of esophageal or esophagogastric junction adenocarcinoma, while more than $90 \%$ of patients with esophageal cancer in Asia, including Japan, have esophageal squamous cell carcinoma (ESCC) [2, 3]. 
Endoscopic resection, including endoscopic mucosal resection and endoscopic submucosal dissection (ESD), is the standard treatment for clinical T1aNOMO ESCC due to a potentially low risk of nodal metastasis $[4,5]$, while esophagectomy is regarded as the standard treatment for clinical T1bNOMO ESCC because of $20-40 \%$ of lymph node metastasis [6, 7]. Although the 3-year overall survival (OS) in patients with clinical T1NOMO (CT1NOMO) ESCC who underwent esophagectomy is over $80 \%[6,8,9]$, postoperative complications and declined quality of life after esophagectomy still remain major problems; therefore, less-invasive procedures have been desired as alternative treatments.

Chemoradiotherapy (CRT) showed promising efficacy in patients with cT1NOMO ESCC in the Japan Clinical Oncology Group (JCOG) 9708, with a complete response rate of $87.5 \%$ and a 4-year survival rate of $80.5 \%$ [10]. More recently, the JCOG0502 demonstrated non-inferiority of CRT to esophagectomy in patients with CT1 NOMO ESCC in the non-randomized part (5-year OS rate: $85.5 \%$ in CRT versus $86.5 \%$ in esophagectomy) [11]. Therefore, CRT could be considered one of the standard treatment options for patients with CT1NOMO ESCC. Therefore, this retrospective study aimed to assess whether CRT has equivalent survival outcomes compared to esophagectomy in patients with cT1N0M0. In addition, we also investigated the safety and efficacy of CRT and esophagectomy in elderly patients, who were not eligible to clinical trials.

\section{Methods}

\section{Patients}

This study included patients with CT1NOMO ESCC who underwent esophagectomy or CRT as the first-line treatment at the National Cancer Center Hospital East between January 2009 and December 2017. The clinical tumor stages were determined by these modalities: Upper gastrointestinal endoscopy and computed tomography (CT) were performed for all patients, while endoscopic ultrasonography and positron emission tomography were performed optionally. In this study, an "elderly patient" was defined as a patient aged 75 years or older.

The main exclusion criteria were as follows: 1) tumor location at the cervical esophagus or abdominal esophagus; 2) prior treatment for ESCC, except those who were finally diagnosed with pathological T1amuscularis mucosae or shallower invasion without lymphovascular invasion after endoscopic resection for other lesions; 3 ) synchronous or metachronous multiple cancers, except stage I, within the previous 5 years. Patients with medical comorbidities were not excluded from this study.

\section{Treatment}

Esophagectomy included open or thoracoscopic radical esophagectomy with or without three-field lymphadenectomy. Transhiatal esophagectomy was also permitted. CRT, comprising radiotherapy and concurrent chemotherapy, mainly consisted of 5-fluorouracil (FU) and platinum. Patients were treated 5 days per week at $1.8 \mathrm{~Gy} /$ day in 28 fractions (total $50.4 \mathrm{~Gy}$ ) or at $2 \mathrm{~Gy} /$ day in 30 fractions (total $60 \mathrm{~Gy}$ ) with megavoltage equipment ( $\geq 6 \mathrm{MV}$ ) using a multiple-field technique. In Patients treated with $50.4 \mathrm{~Gy}$, 
the clinical target volume (CTV) included the primary tumor and regional lymph nodes (LNs) with a $2 \mathrm{~cm}$ craniocaudal margin of the primary tumor. Planning target volume (PTV) was defined as CTV plus a 1-2 $\mathrm{cm}$ margin in the craniocaudal direction and $0.5-1 \mathrm{~cm}$ margin in the lateral direction. After treatment with $41.4 \mathrm{~Gy}$ to the PTV, a booster dose of $9.0 \mathrm{~Gy}$ was administered to the primary tumor. In patients treated with $60 \mathrm{~Gy}$, CTV included the primary tumor with a $2 \mathrm{~cm}$ craniocaudal margin. PTV was defined the same as above. Irradiation of $60 \mathrm{~Gy}$ to the PTV was performed without prophylactic irradiation for regional LNs.

\section{Clinical outcomes and statistical analysis}

OS was defined as the time from the initiation date of treatment to death, and progression-free survival (PFS) was defined as the time from the initiation date of treatment to recurrence or death. In the CRT group, esophageal preservation period was defined as the time from the initiation date of treatment to salvage surgery or death. Recurrence was defined as distant metastasis, LN metastasis, or local recurrence that needed surgical resection for cure. Local recurrence that can be cured with endoscopic treatment was not defined as a recurrence in this study. The adverse events of esophagectomy and CRT were evaluated with the Clavien-Dindo classification version 2.0 [12] and the Common Terminology Criteria for Adverse Events version 5.0. Early toxicity and hematological adverse events of the CRT group in particular were defined as events observed between the initiation day of treatment and 30 days after the end of treatment, and late toxicity was defined as events observed more than 30 days after the end of treatment.

Patients' clinical information was collected by retrospectively reviewing their medical records. This study was conducted in accordance with the Ethical Guidelines for Medical Research Involving Human Subjects and was approved by the Ethical Review Committee of the National Cancer Center Hospital East (2017 120).

The Fisher's exact test or the chi-square test was performed to compare categorical variables and the student t-test was performed to compare continuous variables. Survival curves were estimated using the Kaplan-Meier method and differences between the groups were tested by the log-rank test. Hazard ratios were estimated using the Cox proportional hazards model. All $P$ values $<0.05$ were considered statistically significant and statistical analyses were performed using the IBM SPSS statistical software package (version 23.0 for Mac, IBM Japan Ltd., Tokyo, Japan).

\section{Results}

\section{Characteristics of Patients}

This study included 120 patients in the esophagectomy group and 36 patients in the CRT group. Among them, 21 and 11 elderly patients were included in the esophagectomy and CRT groups, respectively (Supplementary Fig. 1). The esophagectomy and CRT groups had similar patients' characteristics, except clinical tumor depth (SM2/3: 84.2\% in the esophagectomy group vs. $61.1 \%$ in the CRT group, $\mathrm{P}=0.005$ ) (Table 1). Synchronous stage 0 or I ESCC at diagnosis was detected in 18 patients in the esophagectomy 
group (15.0\%) and five patients in the CRT group (13.9\%). Only two patients in the esophagectomy group had history of previous treatment with endoscopic resection for pathological T1a ESCC. 
Table 1

Baseline patient characteristics

All patients $(n=156)$

Elderly patients $(n=32)$

\begin{tabular}{|c|c|c|c|}
\hline $\begin{array}{l}\text { Esophagectomy } \\
(\mathrm{n}=120)\end{array}$ & $\begin{array}{l}\text { CRT } \\
(n=36)\end{array}$ & $P$ values & $\begin{array}{l}\text { Esophagectomy } \\
(n=21)\end{array}$ \\
\hline $1, \%$ & & & $n \%$ \\
\hline
\end{tabular}

$\mathrm{n}, \% \quad \mathrm{n}, \%$

n, \%

Age, year

(median, range)

Sex

Male

Female

ECOG PS

0

1

2

Tumor location

$67(46-87)$

96 (79.7)

$24(20.3)$

70
$(38-$
$85)$

32

(88.8)

$4(11.1)$

$0.360 * \quad 78(75-87)$

77

$(75-$

85)

9

$1(4.8)$

2

$105(87.5)$

$11(9.2)$

4 (3.3)

$1(2.8)$

2 (5.6)

33

(91.7)

$0.391^{\star \star \star} \quad 16(76.1)$

$5(23.8)$

0

10

(90.9)

1

0

$4(11.1) \quad 0.067^{\star \star \star} \quad 3(14.3)$
Ut

Mt

Lt

26

(72.2)

47 (39.2)

$6(16.7)$

10 (47.6)

$8(38.1)$

(72.7)

2

Clinical tumor depth

19 (15.8)

$101(84.2)$

14

(38.9)

MM/SM1

SM2/SM3

Horizontal location

74 (61.7)

46 (38.3)

$\leq 3 / 4$

$>3 / 4$

Intramural metastasis
22

(61.1)

17

(47.2)

19

(52.8)

2 (5.6) $\quad 0.326^{\star \star} \quad 1(4.8)$
6

$19(90.5)$

5

5

$6(28.6)$

6

1

*Student t-test, ${ }^{\star *}$ Fisher's exact test, ${ }^{\star} \star \star$ chi-square test, Esophagectomy vs. CRT in all patients. CRT: Chemoradiotherapy; ECOG PS: Eastern Cooperative Oncology Group performance status; ESCC: esophageal squamous cell carcinoma; Lt: lower thoracic esophagus; MM: muscularis mucosae; Mt: Middle thoracic esophagus; SM: submucosa; Ut, upper thoracic esophagus. 


\begin{tabular}{|c|c|c|c|c|c|}
\hline \multicolumn{4}{|c|}{ All patients $(n=156)$} & \multicolumn{2}{|c|}{ Elderly patients $(n=32)$} \\
\hline $\begin{array}{l}\text { History of treatment for } \\
\text { ESCC }\end{array}$ & $2(1.7)$ & 0 & $1.000 \star \star$ & 0 & 0 \\
\hline $\begin{array}{l}\text { Synchronous other ESCC } \\
\text { at diagnosis }\end{array}$ & $18(15.0)$ & $5(13.9)$ & $1.000 * \star$ & $2(9.5)$ & 0 \\
\hline $\begin{array}{l}\text { Synchronous other cancer } \\
\text { at diagnosis }\end{array}$ & 16 (13.3) & $3(8.3)$ & $0.567^{\star \star *}$ & $3(14.3)$ & 0 \\
\hline Comorbidity & $57(47.5)$ & \multirow{2}{*}{$\begin{array}{l}13 \\
(36.1)\end{array}$} & $0.256^{\star \star}$ & $16(76.2)$ & \multirow{2}{*}{$\begin{array}{l}6 \\
(54.5)\end{array}$} \\
\hline Cardiovascular & $13(10.8)$ & & $0.523^{\star *}$ & $2(9.5)$ & \\
\hline Respiratory & $16(13.3)$ & $\begin{array}{l}2(5.6) \\
5(13.9)\end{array}$ & $1.000 * \star$ & $4(19.0)$ & $\begin{array}{l}2 \\
(18.2)\end{array}$ \\
\hline & $9(7.5)$ & \multirow[t]{2}{*}{$2(5.6)$} & $1.000 * \star$ & $2(9.5)$ & $\begin{array}{l}1 \\
(9.1)\end{array}$ \\
\hline Brain disease & & & & & 0 \\
\hline $\begin{array}{l}\text { *Student t-test, }{ }^{\star \star} \text { Fisher's e } \\
\text { Chemoradiotherapy; ECOG } \\
\text { esophageal squamous cell } \\
\text { Middle thoracic esophagus }\end{array}$ & $\begin{array}{l}\text { test, }{ }^{\star * *} \mathrm{C} \\
\text { Eastern C } \\
\text { cinoma; L }\end{array}$ & $\begin{array}{l}\text { t, Esoph } \\
\text { ncology } \\
\text { cic esop }\end{array}$ & $\begin{array}{l}\text { ectomy } \\
\text { roup per } \\
\text { ggus; Mn }\end{array}$ & $\begin{array}{l}\text { CRT in all } p \\
\text { nance statı } \\
\text { huscularis }\end{array}$ & $\begin{array}{l}\text { CRT: } \\
\vdots \\
\text { Mt: }\end{array}$ \\
\hline
\end{tabular}

Table 2

Treatment details in patients with esophagectomy (A) and CRT (B)

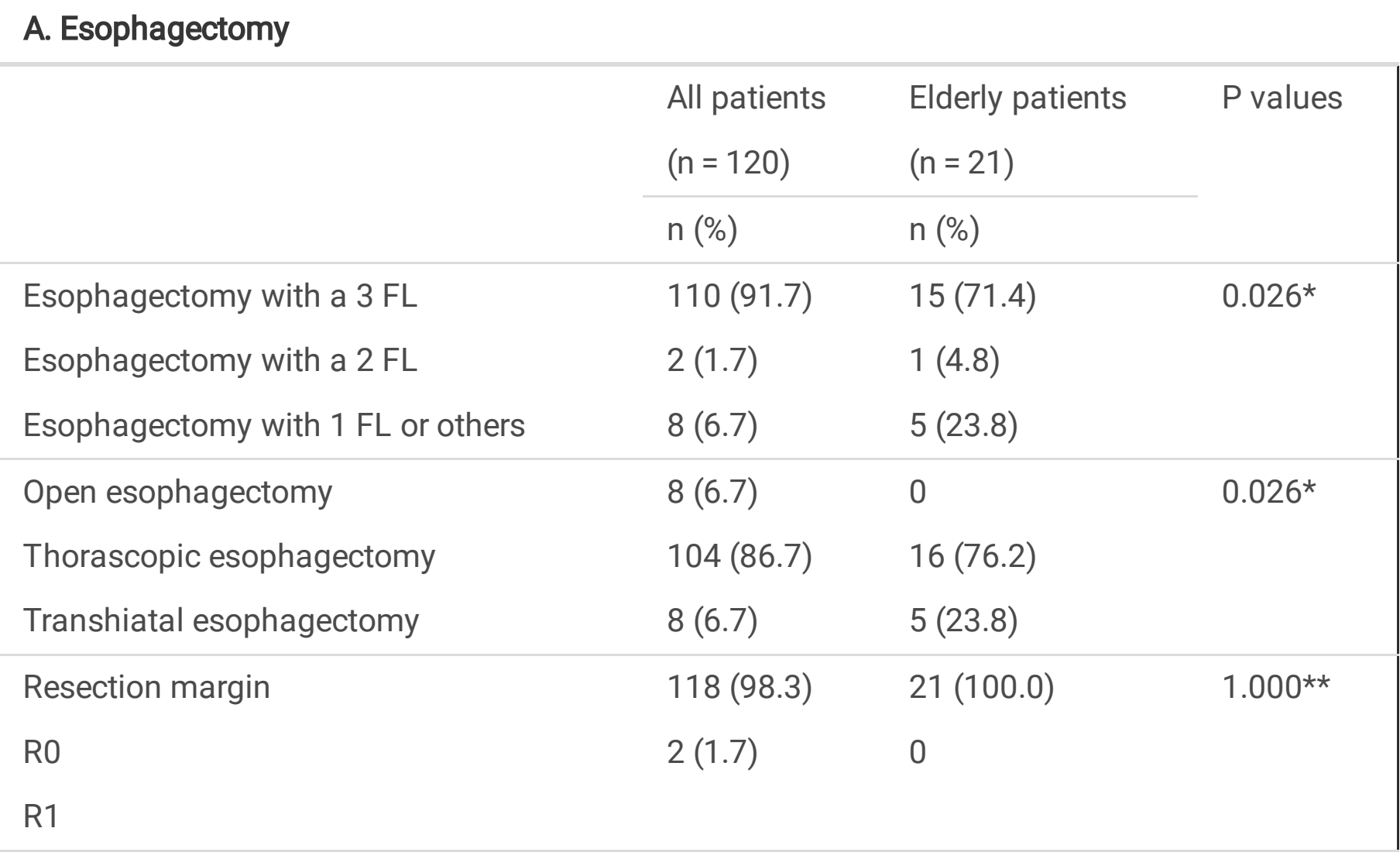

* Chi-square test, ** Fisher's exact test, All patients vs. Elderly patients. FL: field lymphasectomy 
Table 3

Complications in patients with esophagectomy (A) and CRT (B)

\section{B. CRT}

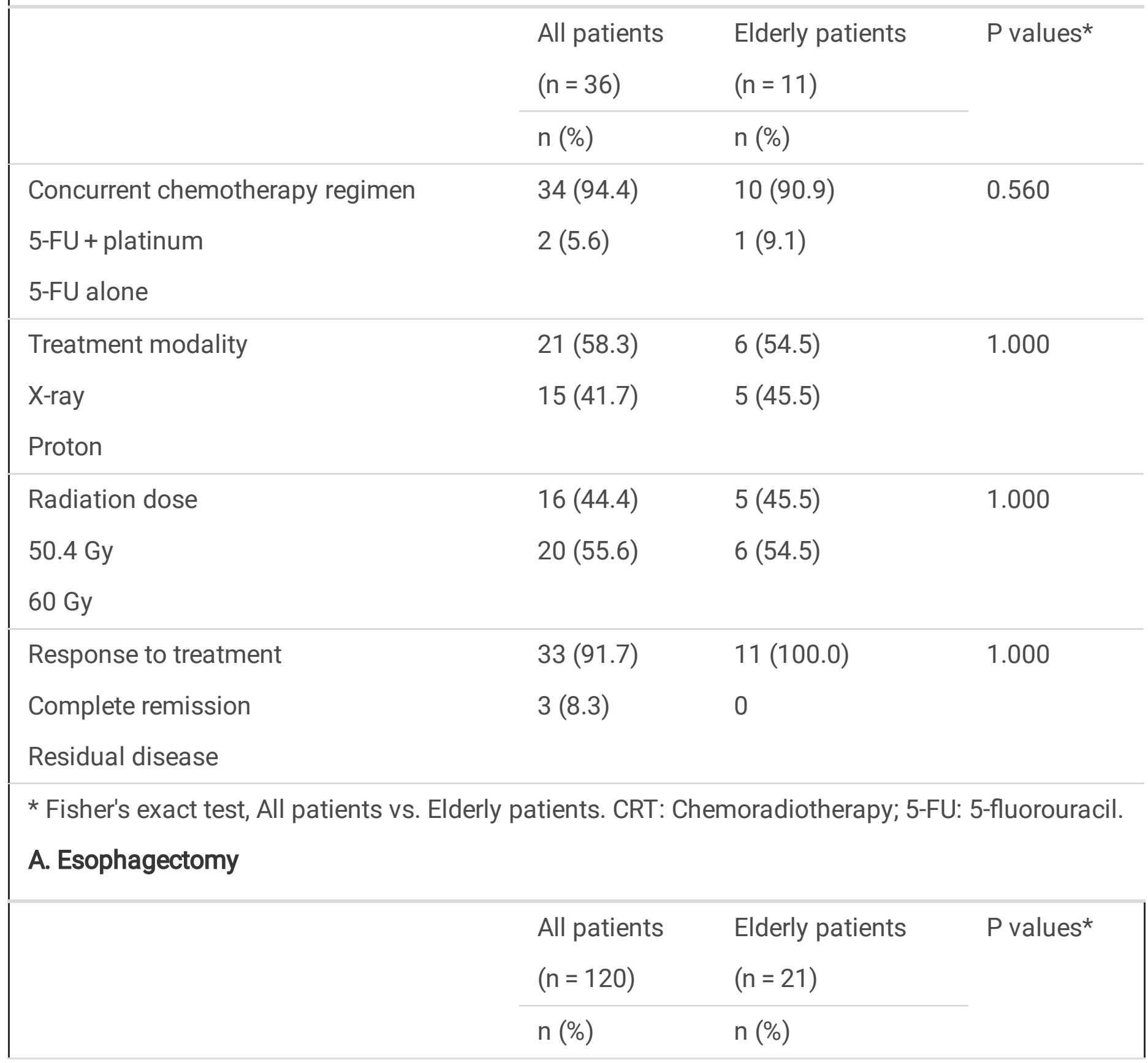

* Fisher's exact test, All patients vs. Elderly patients. RL nerve: Recurrent laryngeal nerve 


\begin{tabular}{|llll|}
\hline B. CRT & & & \\
\hline Early complication & $72(60.0)$ & $14(66.7)$ & 0.634 \\
Any & $22(18.3)$ & $4(19.0)$ & 1.000 \\
An nerve paralysis & $15(12.5)$ & $1(4.8)$ & 0.467 \\
Surgical site infection & $9(7.5)$ & $4(19.0)$ & 0.106 \\
Pneumonia & $7(5.8)$ & $3(14.3)$ & 0.171 \\
Lymphorrhea & $6(5.0)$ & $1(4.8)$ & 1.000 \\
Chylothorax & $4(3.3)$ & 0 & 1.000 \\
Ileus & $3(2.5)$ & 0 & 1.000 \\
Bleeding & $2(1.7)$ & 0 & 1.000 \\
Pneumothorax & $2(1.7)$ & $1(4.8)$ & 0.386 \\
Late complication & $31(25.8)$ & $8(38.1)$ & 0.292 \\
Any & $26(21.7)$ & $6(28.6)$ & 0.572 \\
Esophageal stenosis & $3(2.5)$ & 0 & 1.000 \\
Ileus & $2(1.7)$ & 0 & 1.000 \\
Bleeding & & & \\
\hline * Fisher's exact test, All patients vs. Elderly patients. RL nerve: Recurrent laryngeal nerve & \\
\hline
\end{tabular}

Treatment details

Treatment details and pathological outcomes in patients who received esophagectomy are shown in Table 2A and Supplementary Table 1. Esophagectomy with three-field lymphadenectomy was performed in 110 patients $(91.7 \%)$. Post-operative histological findings revealed that 86 patients $(71.7 \%)$ had pathological T1NOM0 disease, and 34 patients $(28.3 \%)$ had a pathological non-T1NOMO disease. Thirtytwo patients $(26.7 \%)$ had LN metastasis, and six patients (5.0\%) had deeper tumor depth (four patients had both LN metastasis and deeper tumor). Although most of the patients achieved complete resection (R0), two patients (1.7\%) had microscopic residual disease (R1). After esophagectomy, six patients with non-T1N0M0 disease underwent adjuvant chemotherapy.

Treatment details in the CRT group are shown in Table 2B. Concurrent chemoradiotherapy was performed for all patients. Thirty-four patients $(94.4 \%)$ received the 5 -FU plus platinum combination regimen. In addition, 21 patients $(58.3 \%)$ received $X$-ray therapy, and 15 patients $(41.7 \%)$ received proton beam therapy. Total doses of 60 Gy or Gy equivalents and 50.4 Gy were delivered to 20 patients and 16 patients, respectively. Post-treatment endoscopic evaluation showed that 33 patients $(91.7 \%)$ achieved 
complete remission, while three patients (8.3\%) had residual disease. All three patients with residual diseases underwent additional salvage treatment (ESD in two patients and esophagectomy in one patient).

Safety

Post-operative complications in the esophagectomy group are shown in Table 3A. Seventy-two patients $(60.0 \%)$ had early complications; the main common complications were recurrent laryngeal nerve paralysis (22 patients, $18.3 \%$ ), anastomotic leakage (15 patients, $12.5 \%$ ), and surgical site infection (9 patients, 7.5\%). Esophageal stenosis was the most frequent (26 patients, $21.7 \%$ ) late complication. There was no post-operative mortality within 30 days of esophagectomy.

Adverse events in the CRT group are shown in Table 3B and Supplementary Table 2. Most of the patients experienced hematologic adverse events; particularly, grade 3 or higher leucopenia and neutropenia were observed in nine patients (25.0\%). Esophagitis was observed in 31 patients (86.1\%); it was the most common early toxicity. Radiation-related late toxicities including pleural effusion, pericardial fluid accumulation, and irradiation pneumonitis were observed in six (16.7\%), four (11.1\%), and three (8.3\%) patients, respectively.

\section{Efficacy}

With a median follow-up period of 71.8 months (72.8 months in esophagectomy group and 70.8 months in CRT group) with Kaplan-Meier estimate, the $5 y$-PFS was $77.0 \%$ in the esophagectomy group and $74.4 \%$ in the CRT group, with no significant difference between the two groups $(P=0.48)$ (Fig. 1A). The 5y-OS was $81.5 \%$ in the esophagectomy group and $82.6 \%$ in the CRT group, with no significant difference between the two groups $(P=0.89)(F i g .1 B)$. The results of univariate and multivariate analysis for PFS and OS with clinical factors are shown in supplementary table 4 and 5. Particularly, according to the clinical tumor invasion depth, which was based on clinical factors between the treatment groups, the 5yPFS and OS in patients with CSM2/SM3 disease tend to be worse compared to those with MM/SM1 (5yPFS, $87.9 \%$ vs. $73.4 \%$; 5y-OS, $90.6 \%$ vs. $79.5 \%$ ) (Supplementary Fig. 2). However, even in multivariate analysis, the adjusted hazard ratio of CRT was $1.299(95 \% \mathrm{Cl}[0.627-2.689], \mathrm{p}=0.48)$ for PFS and 1.007 for OS $(95 \% \mathrm{Cl}[0.438-2.315], \mathrm{p}=0.99)$, respectively; there was no significant difference in efficacy between esophagectomy and CRT. In the CRT group, the 3y- and 5y-survival rates with esophageal preservation were $80.4 \%$ and $76.9 \%$, respectively.

Treatments after recurrence

During the follow-up period, 21 patients (17.5\%) in the esophagectomy group and nine patients $(25.0 \%)$ in the CRT group experienced disease recurrence. The details of recurrence sites and additional treatments are shown in Table 4 and Supplementary Table 3. The rates of local recurrence ( $0 \%$ vs. $2.8 \%$ ), regional LN metastasis ( $5.8 \%$ vs. $8.3 \%)$, and distant metastasis ( $11.7 \%$ vs. $13.9 \%$ ) were similar between the esophagectomy and CRT groups; however, bone metastasis $(n=5)$ was observed only in the 
esophagectomy group. Among the 30 patients with recurrence, salvage surgery was performed in three of 21 patients in the esophagectomy group (14.3\%) and five of nine patients in the CRT group (55.5\%), and their recurrence site was regional $\operatorname{LN}(n=4)$, lung $(n=3)$, and local $(n=1)$ recurrence. Salvage CRT was performed in five of 21 patients in the esophagectomy group (23.8\%), and all of them had regional LN recurrence. In each group, palliative chemotherapy was selected in four patients who had recurrence in distant LN, lung, and liver. Particularly, nine of 21 patients in the esophagectomy group received best supportive care (BSC) alone after recurrence due to their poor general condition. There were no significant differences in the recurrence rates between the two treatment groups; however, BSC alone was selected more frequently in the esophagectomy group $(P=0.029)$.

Table 4

Details about recurrence sites and additional treatments

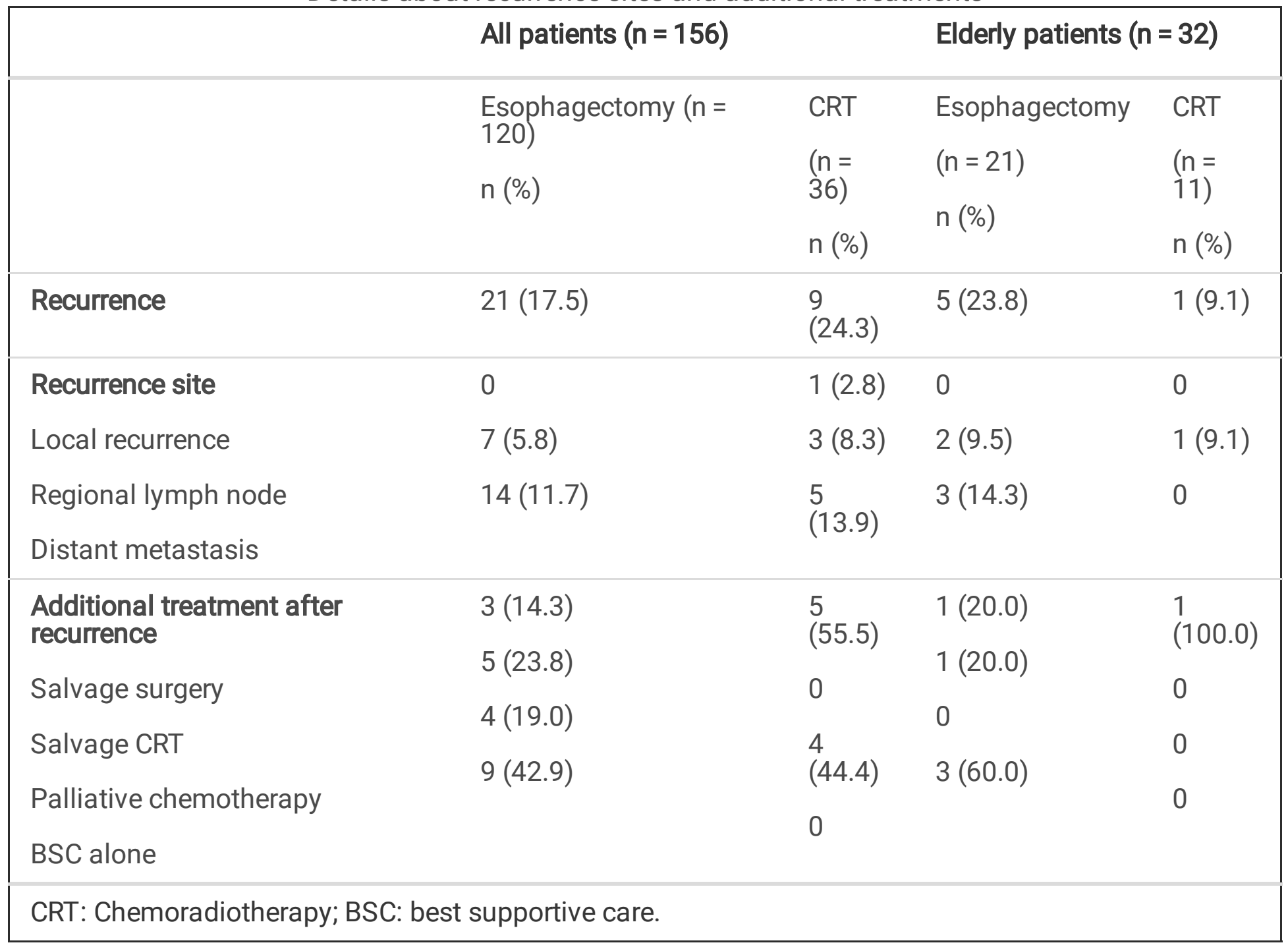

Safety and efficacy in elderly patients

Among a total of 32 elderly patients, 21 patients underwent esophagectomy and 11 patients received CRT. The rate of clinical SM2/3 tumor was higher in esophagectomy group than that in CRT group $(90.5 \%$ vs. $45.5 \%, P=0.01)$. In the esophagectomy group, the rate of three-field lymphadenectomy in elderly patients was lower than that in the total population $(71.4 \%$ vs. $91.7 \%, P=0.026)$ and the rate of 
transhiatal esophagectomy was higher $(23.8 \%$ vs. $6.7 \%, P=0.026)$ (Table $2 A)$. On the contrary, details of treatment modality and radiation doses in the CRT group were similar between elderly patients and all patients (Table 2B). In the safety profile, there were no significant differences in the frequency of postoperative complications in the esophagectomy group (Table $3 \mathrm{~A}$ ) and the frequency of adverse events in the CRT group between all patients and elderly patients, although pleural effusion after CRT tended to be higher in elderly patients (Table 3B). The 5y-PFS was $72.3 \%$ and $81.8 \%(\mathrm{HR} 0,55 ; 95 \% \mathrm{Cl}, 0.14-2.13$ ) and the $5 y-O S$ was $76.9 \%$ and $81.8 \%(\mathrm{HR} \mathrm{0,68;95 \%} \mathrm{Cl}, 0.16-2.81)$ in the esophagectomy group and the CRT group, respectively (Fig. 2). After adjusting the clinical tumor depth on Cox regression analysis, there were still no statistical differences between the esophagectomy and CRT groups in the survival outcomes; PFS, adjusted $\mathrm{HR}=0.34,95 \% \mathrm{Cl}[0.061-1.856], \mathrm{p}=0.211 ;$ OS, adjusted $\mathrm{HR}=0.32,95 \% \mathrm{Cl}[0.044-2.243], \mathrm{p}=$ 0.249 .

\section{Discussion}

We retrospectively evaluated the safety and efficacy of esophagectomy and CRT in patients with CT1NOMO ESCC, including elderly patients, using real-world data. There were no significant differences in the safety and efficacy between the esophagectomy and CRT groups, irrespective of age.

Both National Comprehensive Cancer Network (NCCN) guidelines and European Society of Medical Oncology (ESMO) Clinical Practice Guidelines recommend esophagectomy as a standard of care for cT12NO ESCC disease, and chemoradiation is only referred in locally advanced disease, not in cT1-2NO disease, in these guidelines $[13,14]$. Notably, the Japanese guideline mentions that chemoradiation is a treatment option in patients with CT1NOMO disease who are not fit for or unwilling to undergo esophagectomy. Indeed, the similar OS of esophagectomy and CRT found in the JCOG0502 supports the recommendations in the Japanese guideline, despite the non-randomized findings.

In this study, the PFS in the CRT group seems to be slightly worse than that of the esophagectomy group, although it was not statistically significant $(\mathrm{HR}=1.26,95 \% \mathrm{Cl}[0.64-2.56])$, which was consistent with the results of JCOG0502. Notably, local recurrence or regional lymph node recurrence were observed in four of nine patients with recurrent disease; then, all four patients underwent salvage surgery (supplementary table 3). These facts may lead to a similar OS between the treatment groups. If patients choose CRT for CT1NOMO ESCC, careful follow-up is important to ensure that the timing for salvage surgery for recurrent disease is not missed.

We also assessed the efficacy in elderly patients who underwent esophagectomy or CRT. A large-scale cohort study with the Japanese National database of Hospital-Based Cancer Registries found that survival outcomes of CRT were comparable to those of esophagectomy in elderly patients with clinical stage I esophageal cancer [15]. Although there was a selection bias because elderly patients included in our study were all clinically fit for esophagectomy or CRT, our results were consistent with those in the cohort study, showing the comparable efficacy of CRT and esophagectomy in elderly patients with cT1NOMO ESCC. In addition, our study showed acceptable complications in elderly patients with CRT, 
although most of them underwent CRT with concurrent doublet chemotherapy. These findings indicate that CRT is a promising optional treatment for patient of all ages with cT1NOMO ESCC.

This study has some limitations. First, this was a single-center, retrospective study. Patients' selection bias due to the retrospective nature, including higher proportion of patients with CSM2/SM3 disease in the esophagectomy group, might have led to decreased 5y-PFS and 5y-OS in the esophagectomy group as compared to the CRT group. However, comparable results in the multivariate analysis support the similar survival outcomes of CRT and esophagectomy in our study. Second, the sample size of this study was small; only 21 and 11 elderly patients were included in the esophagectomy and CRT groups, respectively. Despite these limitations, our findings would help decide the treatment strategy for patients with cT1NOMO ESCC in clinical practice as it is difficult to conduct randomized trials to compare the efficacy of esophagectomy versus CRT.

\section{Conclusion}

Our real-world study showed acceptable safety and favorable efficacy of CRT in patients with cT1NOMO ESCC, including elderly patients. This study confirms that CRT is one of the standard treatment options for patients of all ages with cT1NOMO ESCC in clinical practice.

\section{Abbreviations}

BSC: best supportive care; CRT: Chemoradiotherapy; CT: computed tomography; CTV: clinical target volume; ESCC: esophageal squamous cell carcinoma; ESD: endoscopic submucosal dissection; ESMO: European Society of Medical Oncology; FU: fluorouracil; JCOG: Japan Clinical Oncology Group; LNs: lymph nodes; NCCN: National Comprehensive Cancer Network; OS: overall survival; PFS: progression-free survival; PTV: planning target volume,

\section{Declarations}

\section{Ethics approval and consent to participate}

This study was conducted in accordance with the Ethical Guidelines for Medical Research Involving Human Subjects and was approved by the Ethical Review Committee of the National Cancer Center Hospital East (2017-120).

\section{Consent for publication}

Not applicable

\section{Availability of data and materials}

The datasets generated during and/or analyzed during the current study are available from the corresponding author on reasonable request. 


\section{Competing interests}

TK declare that he receives research funding to institutions from MSD, Ono Pharmaceutical Co., Ltd., Bristol-Myers Squibb, Astellas Amgen BioPharma, Taiho Pharmaceutical, Chugai, Shionogi.

TY declare that he receives grant for out of this study from Olympus, Fujifilm, HOYA Pentax, Rakuten medical. DK, KN, KS, HF, HH, HY, MN, SM, TF, TK and YY declare that they have no competing interests.

\section{Funding}

No funding was received in the publication of this article.

\section{Author contributions}

KS, DK, TK and HF designed the research; HF, HY, SM, TK and KN collected the data; KS analyzed the data; KS and DK wrote the manuscript; HF, HY, SM, DK, TK, YY, MN, HH, TY, TF and TK reviewed and approved the final version of the manuscript.

\section{Acknowledgments}

We would like to thank Daiki Sato, Atshushi Inaba, Keiichiro Nishihara for data collection and Editage (www.editage.com) for English language editing.

\section{References}

1. Bray F, Ferlay J, Soerjomataram I, et al. Global cancer statistics 2018: GLOBOCAN estimates of incidence and mortality worldwide for 36 cancers in 185 countries. CA Cancer J Clin. 2018;68:394424.

2. Castro $C$, Bosetti $C$, Malvezzi M, et al. Patterns and trends in esophageal cancer mortality and incidence in Europe (1980-2011) and predictions to 2015. Ann Oncol. 2014;25:283-90.

3. Christian CA, Melina A, Wei WQ. Epidemiology of esophageal squamous cell carcinoma. Gastroenterology. 2018;154:360-73.

4. Kitagawa Y, Uno T, Oyama T, et al. Esophageal cancer practice guidelines 2017 edited by the Japan esophageal society: part 2. Esophagus. 2019;16:25-43.

5. Ishihara R, Arima M, lizuka T, et al. Endoscopic submucosal dissection/endoscopic mucosal resection guidelines for esophageal cancer. Dig Endosc. 2020;32:452-93.

6. Igaki H, Kato H, Tachimori Y, et al. Clinicopathologic characteristics and survival of patients with clinical Stage I squamous cell carcinomas of the thoracic esophagus treated with three-field lymph node dissection. Eur J Cardiothorac Surg. 2001;20:1089-94.

7. Akutsu $\mathrm{Y}$, Kato $\mathrm{K}$, Igaki $\mathrm{H}$, et al. The prevalence of overall and initial lymph node metastases in clinical T1N0 thoracic esophageal cancer: From the results of JCOG0502, a prospective multicenter study. Ann Surg. 2016;264:1009-15. 
8. Motoori $\mathrm{M}$, Yano $\mathrm{M}$, Ishihara $\mathrm{R}$, et al. Comparison between radical esophagectomy and definitive chemoradiotherapy in patients with clinical T1bNOM0 esophageal cancer. Ann Surg Oncol. 2012;19:2135-41.

9. Park I, Kim YH, Yoon DH, et al. Non-surgical treatment versus radical esophagectomy for clinical T1 N0M0 esophageal carcinoma: a single-center experience. Cancer Chemother Pharmacol. 2014;74:995-1003.

10. Kato H, Sato A, Fukuda H, et al. A Phase II Trial of Chemoradiotherapy for Stage I Esophageal Squamous Cell Carcinoma: Japan Clinical Oncology Group Study (JCOG9708). Jpn J Clin Oncol. 2009;39:638-43.

11. Kato $\mathrm{K}$, Igaki $\mathrm{H}$, Ito $\mathrm{Y}$, et al. Parallel-group controlled trial of esophagectomy versus chemoradiotherapy in patients with clinical stage I esophageal carcinoma (JCOG0502). J Clin Oncol. 2019;37:7-7. (no. 4_suppl).

12. Katayama H, Kurokawa $Y$, Nakamura K, et al. Extended Clavien-Dindo classification of surgical complications: Japan Clinical Oncology Group postoperative complications criteria. Surg today. 2016;46:668-85.

13. Ajani JA, D'Amico TA, Bentrem DJ, et al. Esophageal and esophagogastric junction cancers, version 2.2019, nccn clinical practice guidelines in oncology. J Natl Compr Canc Netw. 2019;17:855-83.

14. Lordick F, Mariette C, Haustermans K, et al. Oesophageal cancer: ESMO Clinical Practice Guidelines for diagnosis, treatment and follow-up. Ann Oncol. 2016;27(suppl 5):v50-7.

15. Motoyama S, Maeda E, lijima K, et al. Does esophagectomy provide a survival advantage to patients aged 80 years or older? Analyzing 5,066 Patients in the National Database of HospitalBased Cancer Registries in Japan. Ann Surg. 2020. doi:10.1097/SLA.0000000000004437.

\section{Figures}


A Progression free survival

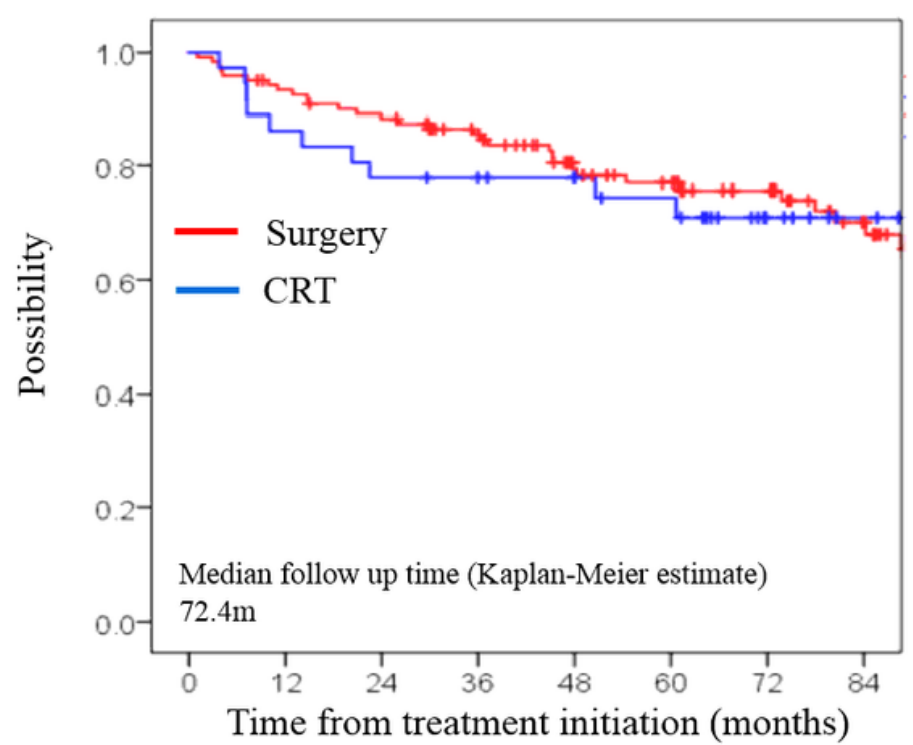

Number at risk

\begin{tabular}{lrrrrrrrr}
$\begin{array}{l}\text { Surgery } \\
\text { CRT }\end{array}$ & 120 & 110 & 103 & 91 & 70 & 61 & 49 & 33 \\
& 36 & 31 & 28 & 26 & 24 & 21 & 10 & 5 \\
\hline & Group & $3 y-P F S$ & $5 y-P F S$ & $\begin{array}{c}\text { HR } \\
(95 \% \mathrm{CI})\end{array}$ & $P$ \\
\hline & Surgery & $85.5 \%$ & $77.0 \%$ & Ref & - \\
\hline & CRT & $77.8 \%$ & $74.4 \%$ & $1.28(0.64-2.56)$ & 0.48 \\
\hline
\end{tabular}

B

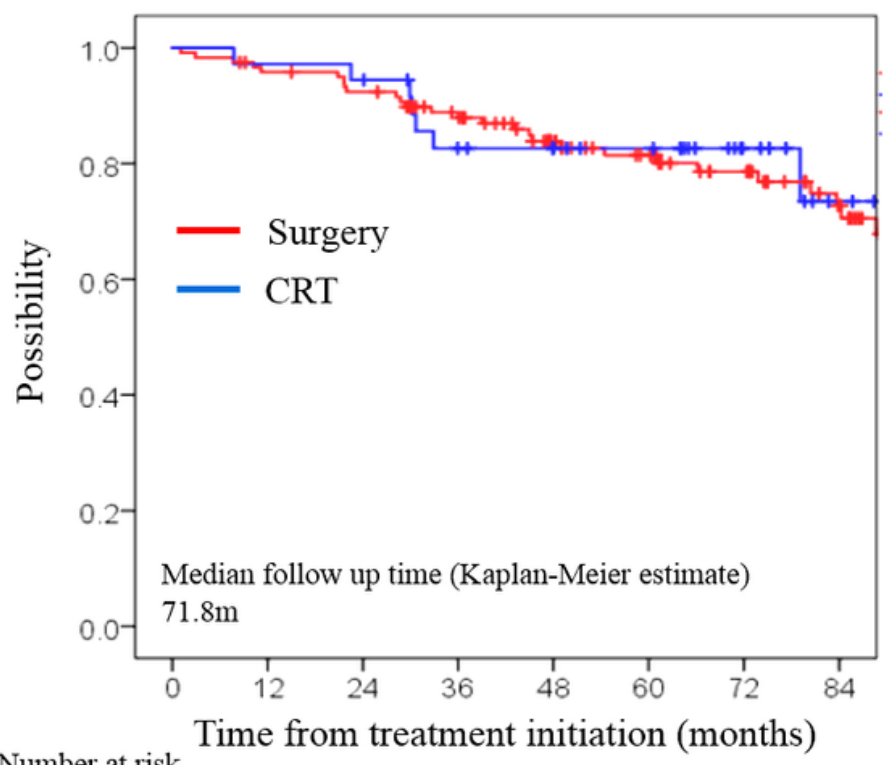

Number at risk

\begin{tabular}{ccccccccc}
$\begin{array}{l}\text { Surgery } \\
\text { CRT }\end{array}$ & 120 & 113 & 108 & 95 & 74 & 63 & 50 & 34 \\
& 36 & 35 & 34 & 27 & 25 & 22 & 12 & 5 \\
\hline & Group & $3 y-O S$ & $5 y-O S$ & $\begin{array}{c}\text { HR } \\
(95 \% \mathrm{CI})\end{array}$ & $P$ \\
\hline & Surgery & $88.9 \%$ & $81.5 \%$ & Ref & - \\
\hline & CRT & $82.6 \%$ & $82.6 \%$ & $1.06(0.48-2.34)$ & 0.89 \\
\hline
\end{tabular}

\section{Figure 1}

PFS in all patients (A) and elderly patients (B) 
A Progression free survival

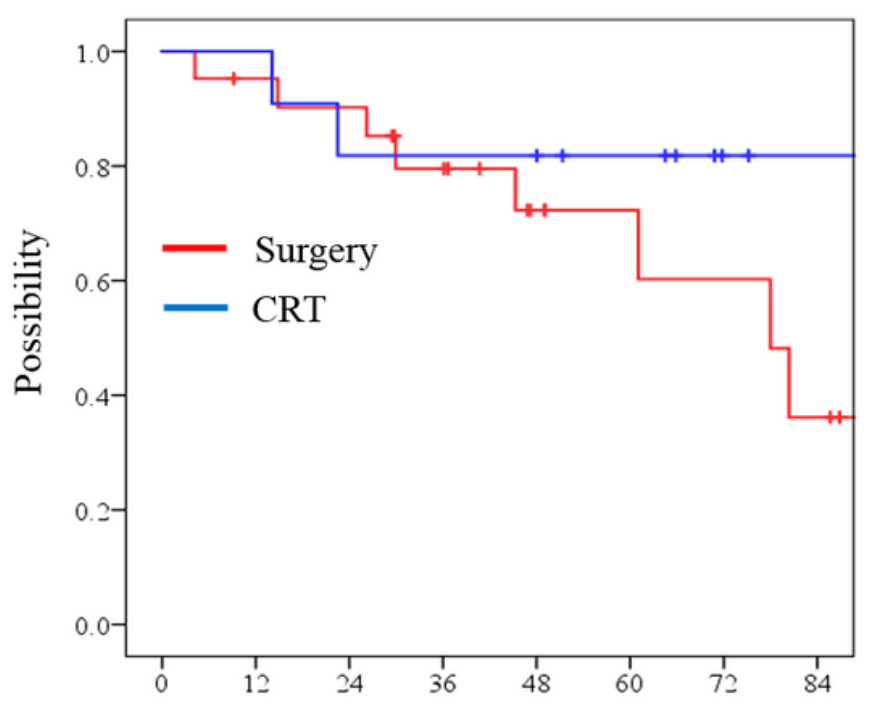

Time from treatment initiation (months)

Number at risk

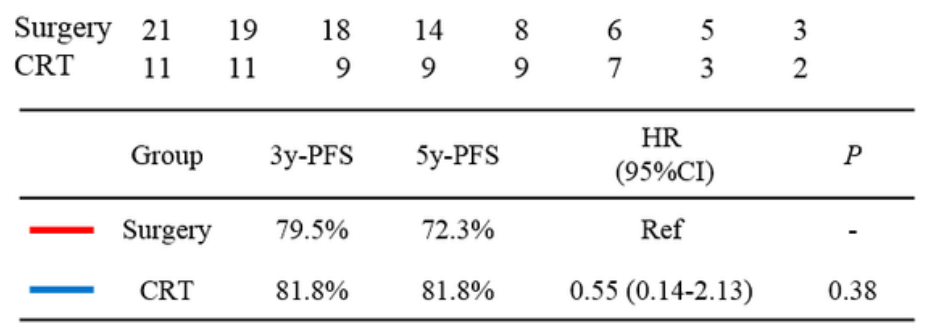

B

Overall Survival

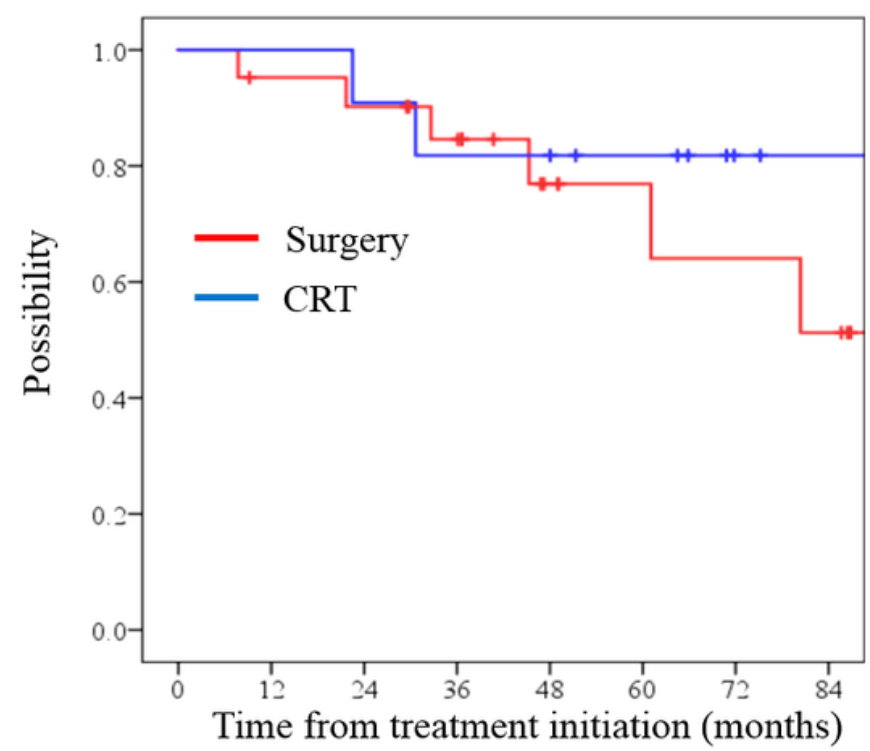

Number at risk

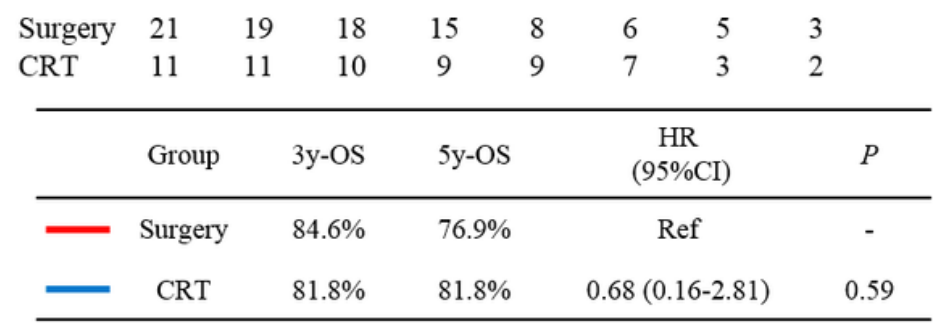

Figure 2

OS in all patients $(A)$ and elderly patients $(B)$

\section{Supplementary Files}

This is a list of supplementary files associated with this preprint. Click to download.

- SupplementaryMaterialver2.6.docx 